\title{
Optimization of Vibration Reduction on the Mast by Active Stay System
}

\author{
by Ya-Jung Lee*, member \\ Wen-Jeng Hsueh**
}

\begin{abstract}
Summary
An investigation of vibration reduction at the top of the mast by active control system is presented. Two typical external excitations, impact and random dynamic load, are considered. For each type of load, a cost function is defined and its optimal law is solved. Besides, an active stay system is designed to offer an active force on the mast.

By comparing the direct stiffness increment and fixed stay auxiliary methods, it is shown that the active stay system has the fastest decay property among these methods for impact induced motion. For random exciting load, it has superior effect of peak suppressed in spectral density response than other two methods and its standard variations much lower than other cases compared. In addition, the lateral translation and slope accelerations at the top of the mast reduced by the active stay system is also the most effective. Numerical examples demonstrate that the active stay system with optimal law is a powerful and feasible scheme for mast vibration reduction.
\end{abstract}

\section{Introduction}

Radar is the primarily visual instrument in warships. For a broad view, high mast is usually used for radar mounting. For good precision in target tracking, the base of the radar is as steady as possible ${ }^{1)-3}$. Thus, the upper bound of the magnitude of vibration is required and specified in military standard ${ }^{4,5}$. To constrain the motion of a high mast at the top, where the radar is located, in stable state under serious loading on the sea, several types of construction design, such as direct stiffness increment and fixed stays auxiliary method, are proposed ${ }^{6,7)}$. In both methods, the design concept is increasing the lateral stiffness of the whole structure to decrease the deflection. It is known that the lateral deflection constraint at the free end of a cantilever structure is of low efficiency by the direct stiffness increment method. In order to maintain the required operating environment of radar, large construction is usually needed. Hence, weight increment and the raising of the center of the gravity of the ship are major disadvantages in this design. In fixed stays auxiliary type, the elongation of the stays and the flexibility of mast body will reduce the efficiency of vibration constraint. Thus, thick and numerous stays are usually required for implementation. In addition, the frequency of the structure motion subjecting to loading will rise

* Professor of Institute of Naval Architecture, National Taiwan University, Taipei, Taiwan.

** Ph. D. student of Institute of Naval Architecture, National Taiwan University, Taipei, Taiwan. when the structure becomes more stiff. Then, accelerations of the response may not be reduced effectively for either type. Unfortunately, those accelerations may feed into the servo loop of the radar system and make the operation performance of radar drop.

In fact, target location is estimated, according to the rotation angle of radar antenna, based on the ship coordinate system. Thus, translation movement of the mast at the location of the radar is acceptable, but the slope of the mast at the radar location should not be changed. Therefore, a concept of vibration suppression of the mast by flexible structure design with active control system, which has been applied to the dynamic control of spacecraft, vehicle, and civil engineering ${ }^{8)-10)}$, is proposed ${ }^{11}$. By appropriate arrangement of the actuator and control action of active force, the system can be designed to suppress the motion of the slope at the radar location regardless the motion of other parts of the mast. Since only local suppression is required, high quality of vibration environment for radar can be easily achieved by low power supply.

In this paper, the optimal design of vibration reduction on the mast by active control system is presented. Two typical external exciting forces on the mast are considered: ( 1 ) impact load—induced by slamming, bomb explosion, and missile launching ; ( 2 ) nondeterministic dynamic load-induced by deck vibration, wind gust, and radar motion. For each type of load, a cost function is defined to measure the effect of the vibration reduction of the mast and power consumption of the active system. Then the minimization of the cost function is analyzed to obtain the optimal control func- 
tion that the actuator should follow. To offer an active force on the mast, an active stay system is designed. The flexibility of the stay and the mast is considered in the dynamic analysis of the system. To demonstrate the effect of this scheme, four types of constructions (original polemast, direct stiffness increment, with fixed stay auxiliary, and with active stay system), subjected to both kinds of load, are computed and compared in numerical examples. Furthermore, the active forces needed for those external loads are computed to understand the practicability of this scheme.

\section{Theory}

\subsection{Governing Equation}

The mast of a ship is considered as a cantilever beam in which the mass of radar is lumped at the top shown in Fig. 1. Distributed on the mast, an external exciting force $f_{e}$ is considered. In order to suppress the motion of the mast, some active controlled forces $f_{a}$ are generated and acted on the mast. One-dimension lateral motion is assumed. The governing equation of the mast with respect to the ship coordinate system is given by ${ }^{12)}$

$$
\begin{aligned}
& m(y) \frac{\partial^{2} x(y, t)}{\partial t^{2}}+c_{e}(y) \frac{\partial x(y, t)}{\partial t} \\
& +\frac{\partial^{2}}{\partial y^{2}}\left(c_{s}(y) I(y) \frac{\partial^{3} x(y, t)}{\partial y^{2} \partial t}\right. \\
& \left.+E(y) I(y) \frac{\partial^{2} x(y, t)}{\partial y^{2}}\right)=f_{e}(y, t)+f_{a}(y, t)
\end{aligned}
$$

in which $m(y)$ is the distributed mass of mast and appendages ; $c_{e}(y)$ is the distributed external damping ; $c_{s}(y)$ is the distributed internal damping; $E(y)$ is the Young's modulus; $I(y)$ is the distributed moment of inertia.

Using the finite element method (FEM) concept, the mast can be discretized. If lateral translation and slope are chosen as the degree-of-freedom (DOF) for each node, there will be $2 n$ DOFs in the discrete model when $\mathrm{n}$ nodes are taken. Thus, the governing equation becomes $2 n$ differential equations expressed in matrix form as

$$
M \frac{d^{2} X}{d t^{2}}+C \frac{d X}{d t}+K X=F_{e}+F_{a}
$$

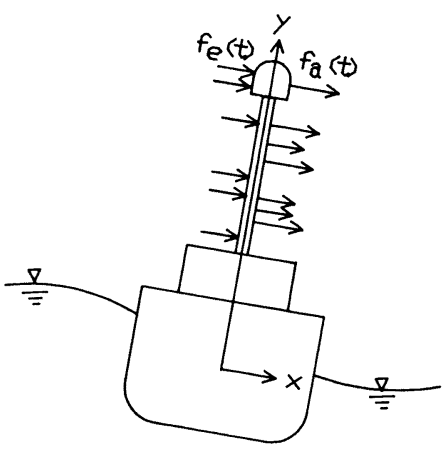

Fig. 1 Configuration of Mast Model Seating on the Ship where $M$ is the mass matrix ; $X$ is the generalized coordinates; $C$ is the damping matrix; $K$ is the stiffness matrix ; $F_{e}$ is the generalized load vector ; and $F_{a}$ is the generalized active force vector. For more effective digital computer analysis, $\mathrm{Eq}(2)$ is converted into a set of $4 n$ by $4 n$ first-order differential equations

$$
\frac{d Z}{d t}=A Z+B_{e} F_{e}+B_{a} F_{a}
$$

where $Z=\left[\begin{array}{c}X \\ d X \\ \hline d t\end{array}\right] ; A=\left[\begin{array}{cc}O_{2 n} & \hat{I}_{2 n} \\ -M^{-1} K & -M^{-1} C\end{array}\right] ; B_{e}=B_{a}$

$$
=\left[\begin{array}{c}
O_{2 n} \\
M^{-1}
\end{array}\right] ; O_{2 n} \text { is } 2 n \text { by } 2 n \text { zero matrix ; } \hat{I}_{2 n} \text { is }
$$

an $2 n$ by $2 n$ identity matrix.

\subsection{Requirement of Optimization}

Intuitively one might think the best choice is that the active forces are equal to the negative external exciting forces to neutralize the load, but in practice, it is not so; because the external excitation is not predictable, accurate active forces instantly generated by actuators are difficult. The vibration induced by the small unpredictable error may accumulate and become enormous for low damping material construction, such as that of a mast.

The aims of most active vibration suppression are as follows: (1) If nonzero initial vibration exists, active forces are provided to decay the vibration as soon as possible. (2) If there are external exciting forces on the mast, some active forces are applied to suppress the motion to minimum.

It is clear that the more effective vibration reduction is wanted, the greater magnitude of active force is needed. However, power supply is limited for any physical system. Some additional conditions are necessary and given as follow: (1) The operation keeps the magnitude of active force as small as possible. (2) One can pay different attention of vibration reduction to the different parts of the system and put different weighting factor on that of the system.

To achieve the aims as above, one performance index is defined by

$$
J=\int_{0}^{\infty}\left(\sum_{i=1}^{4 n}\left(q_{i} z_{i}^{2}\right)+\sum_{j=1}^{p}\left(r_{j} f_{a_{j}}^{2}\right)\right) d t
$$

where $p$ is the number of actuator ; $q_{i}$ is a nonnegative value ; $r_{j}$ is a positive value ; $z_{i}$ is the $i$ th element of $Z$; $f_{a}$ is the $j$ th active force. The object is to find the optimal active force for which the performance index is minimum.

\section{3 Optimization for Impact Load}

If external excitation is an impact load, the initial condition of the state, $Z(0)$, is nonzero. By linear algebra, it can be proven that the minimization of such a performance index is a quadratic form given by ${ }^{13)}$

$$
J_{\min }=Z^{T}(0) P(0) Z(0)
$$

where $P(0)$ is a symmetric matrix. By using the Hamilton-Jacobi equation, It is shown that the matrix $P$ is satisfied the matrix Riccati equation

$$
P A+A^{T} P-P B_{a} R^{-1} B_{a}^{T} P+Q=0
$$




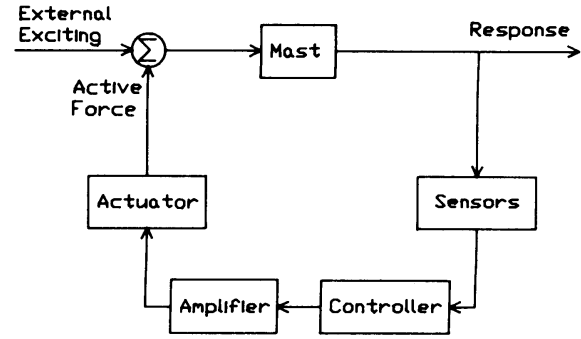

Fig. 2 Flow Chart of Active Control System

where $Q$ is a diagonal matrix with diagonal element $q_{i}$; $R$ is a diagonal matrix with diagonal element $r_{j}$. Then, the optimal active force, $F_{a_{\text {opt }}}$ is uniquely determined by

$$
F_{a_{\text {opt }}}(t)=-G Z(t)
$$

where

$$
G=R^{-1} B_{a}^{T} P
$$

From $\mathrm{Eq}(7)$, it is known that the optimal force is a linear function of displacement and velocity of the response. In other words, putting active control force on the mast is similar to adding a damper and stiffener on it. Hence, the response of the mast and the active device are linked and form a closed-loop system shown in Fig. 2. In order to ensure the stability of the closedloop system, it is necessary that the pair $[A, D]$ is completely observable, where $D$ is any matrix such that

$$
D D^{T}=Q
$$

One of the most general methods to check the observability of a system is to find the rank of the observability matrix $U_{0}$. The system is observable, if the rank of $U_{0}$ is equal to $4 n$, the matrix $U_{0}$ is defined by

$$
U_{0}=\left[D^{T}(D A)^{T}\left(D A^{2}\right)^{T}\left(D A^{3}\right)^{T} \cdots\left(D A^{2 n-1}\right)^{T}\right]^{T}
$$

By substituting Eq. ( 7 ) into Eq. $(3)$, the governing equation of the closed-loop system becomes

$$
\frac{d Z}{d t}=A_{0} Z+B_{e} F_{e}
$$

where

$$
A_{0}=A-R^{-1} B_{a}^{T} P
$$

\section{4 Optimization for Random Load}

In nature, the external exciting force is usually an nondeterministic dynamic load. For convenience, it is assumed that the load is stationary white Gaussian random process and the initial states $Z(0)$ is a random variable, which is Gaussian and independent of the load, given by

$$
\begin{aligned}
& E\left\{F_{e}(t) F_{e}^{T}(\tau)\right\}=W_{f} \delta(t-\tau) \\
& E\left\{Z(0) Z^{T}(0)\right\}=W_{z} \\
& E\left\{F_{e}(t) Z^{T}(0)\right\}=0_{4 n}
\end{aligned}
$$

where $W_{f}$ is the intensity of white noise of $F_{e}(t), W_{z}$ is the autocorrelation matrix of $Z(0), \delta$ is the Dirac delta function. From the assumption of a linear system, one knows that the responses of the mast are also random processes. Thus, the performance index of the vibration reduction is defined by

$$
J=E\left\{\int_{0}^{\infty}\left(\sum_{i=1}^{4 n}\left(q_{i} z_{i}^{2}\right)+\sum_{j=1}^{p}\left(r_{j} f_{a_{j}}^{2}\right)\right) d t\right\}
$$

By stochastic theory, the minimized value of $J$ can be derived and given by ${ }^{13)}$

$$
J_{\min }=\operatorname{tr}\left\{B_{e} V B_{e}^{T} P\right\}
$$

where $\operatorname{tr}$ is the trace function, and $P$ is the solution of the Riccati equation in $\mathrm{Eq}(6)$. It is surprising that the optimal control force in this case is just equal to $\mathrm{Eq}(7)$, the optimal force for impact load. The study indicates that optimal control law of the system is identical for either the impact or random exciting load. To guarantee the stability of the closed-loop system, the pair [ $A$, $D]$ of the system must be completely observable, which is similar to that of the system by impact load.

\section{5 Active Stay System}

Consider a mast, with a radar located at the top as shown in Fig. 1. It is known that if the motion of the whole mast needs to be suppressed, actuators should be installed at each node of the discrete structure model,. Hence, enormous actuators are generally required for enormous elements in FEM modeling. It is clear that full-node action is infeasible for consideration of economy and reliability. Since reducing slope displacement of the mast at the top is the object of our active control system, it can be designed to control the movement at this DOF. Thus, only a pair of stays are sufficient and adopted herein. Stays transfer the active force to the mast from the actuator which is located on the deck. In addition, several sensors are located on the mast to measure motion. The results are used for calculating the required active force by control law. The arrangement of active stay system is shown in Fig. 3.

If the controlled output of the actuator is the stroke displacement, the elasticity of the stay must be considered. Thus, the active force on the mast is a lateral force, which is a function of the stroke displacement, $u(t)$, and lateral displacement of the mast at the location of the fastened stay.

$$
f_{a}(t)=2 \frac{k_{w}}{l_{w}} \sin (\theta)(u(t)-x(y, t) \sin (\theta)) \delta(y-a)
$$

where $k_{w}$ is the stiffness per unit length of the stays, $l_{w}$

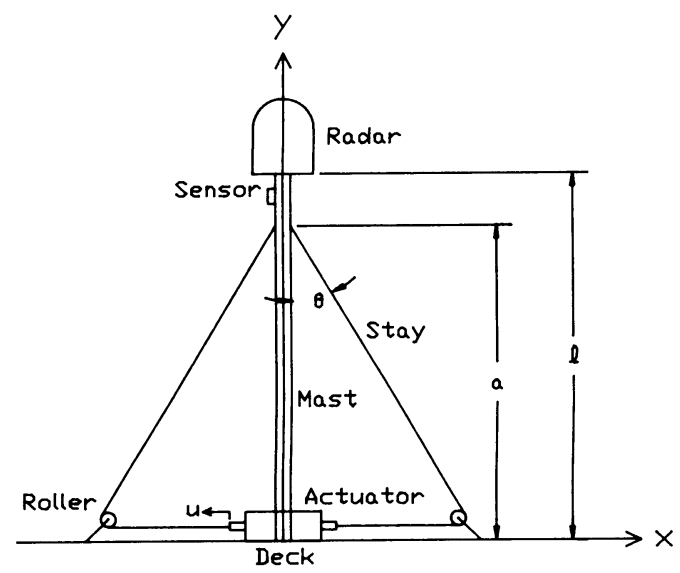

Fig. 3 Arrangement of Active Stay System 
is the length of stays, $a$ is the $y$ coordinate of the stay fastened, $\theta$ is the angle between the stay and the mast.

Substituting Eq. (18) into Eq. (1), it indicates that Eq.

(3) can be rewritten by

$$
\frac{d Z}{d t}=\hat{A} Z+B_{e} F_{e}+\hat{B}_{a} u
$$

where

$$
\begin{aligned}
& \widehat{A}=A+B_{a} K_{w_{1}} \\
& \widehat{B}_{a}=B_{a} K_{w_{2}} \\
& K_{w_{1}}(1,1)=-\frac{2 k_{w}}{l_{w}} \sin ^{2}(\theta), K_{w_{1}}(i, j)=0 \text { for otherwise } \\
& K_{w_{2}}(1)=2 \frac{k_{w}}{l_{w}} \sin (\theta), K_{w_{2}}(i)=0 \text { for otherwise }
\end{aligned}
$$

\section{6 Statistics of Response}

For the linear system assumption, the output of the system is given by the convolution integral

$$
Z(t)=\int_{0}^{\infty} H(\tau) F_{e}(t-\tau) d \tau
$$

where $H$ is the impulse response function. By taking the Fourier transform, the relationship between input and output can be expressed in frequency domain

$$
\widehat{Z}(\omega)=\hat{H}(\omega) \widehat{F}_{e}(\omega)
$$

where $\hat{Z}(\omega), \hat{H}(\omega)$, and $\hat{F}_{e}(\omega)$ are the Fourier transform of $Z(t), H(t)$, and $F_{e}(t)$. For the system considered as Eq. (11), the frequency response function is derived as

$$
\hat{H}(\omega)=\left(i \omega \hat{I}_{4 n}-A_{0}\right)^{-1} B_{e}
$$

where $i=(-1)^{1 / 2}$ and $\hat{I}_{4 n}$ is a $4 n$ by $4 n$ identity matrix. By the stochastic theory, it is known that the spectral density function of $Z, S_{z}(\omega)$, is given by

$$
S_{z}(\omega)=\hat{H}^{*}(\omega) \hat{H}(\omega) S_{f_{e}}(\omega)
$$

where $\hat{H}^{*}(\omega)$ is the complex conjugate of $\hat{H}(\omega), S_{f_{e}}(\omega)$ is the spectral density function of the load $F_{e}(t)$. The standard deviation of $Z$ can be derived by integrating the Eq. (21) and taking the square root ; that is,

$$
\sigma_{z}=\left[\int_{-\infty}^{\infty} S_{z}(\omega) d \omega\right]^{1 / 2}
$$

In similar procedures, the spectral density and the standard deviation of the control force can be obtained.

\section{Numerical Results}

An aluminium alloy tube structure of length $15 \mathrm{~m}$ and having circular cross-section of outside diameter $0.85 \mathrm{~m}$, and a thickness of $0.01 \mathrm{~m}$, is considered as the mast model in the example. Assume that the Young's modulus and the density of the material are $E=6.85 \times$ $10^{10} \mathrm{~N} / \mathrm{m}^{2}$, and $\rho_{m}=2.7 \times 10^{3} \mathrm{~kg} / \mathrm{m}^{3}$. For comparing the effect of vibration reduction of the mast with active stay system, four types of construction are analyzed: (1) original type, polemast without auxiliary; (2) stayed type, polemast with fixed stay auxiliary; (3) stiffened type, with 10 times the EI of the original type ; (4) active type, with the active stay system. The mast is discretized by dividing it into two segments of equivalent length and choosing the lateral translation and slope displacements of each node as the DOF to be considered, shown in Fig. 4. The consistent mass and consistent stiffness methods are used for constructing

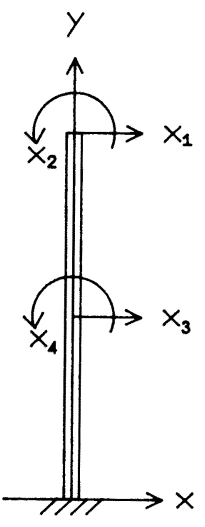

Fig. 4 Discretized Structure Model Used for Example

the mass and stiffness matrix of each element of the discrete model. Rayleigh damping with damping ratio $=0.01$ for each mode is assumed. In the stayed and the active stay types, stays are connected at the top of mast with $\theta=30^{\circ}$ and the elastic stiffness per meter of the stay is chosen $k_{w}=5 \times 10^{7} \mathrm{~N}$. The mass and the mass moment of inertia of the radar are $m_{r}=500 \mathrm{~kg}$ and $I_{r}=$ $500 \mathrm{~kg}-\mathrm{m}^{2}$. Sensors are distributed at the nodes of the discrete model to measure the lateral translation and slope motions.

In the active stay type, since the major object to be controlled is the slope at the top, this state must be weighted more heavy. In addition, to alleviate the acceleration of both lateral translation and slope motions at the top, the states with respect to the velocity of these DOF is weighted more lightly. Hence, the weighting of the performance index are chosen in the illustration as : $q_{22}=5 \times 10^{3}, q_{55}=1, q_{66}=100, q_{i j}=0$ for otherwise, and $r_{11}=10^{4}$. By solving the Riccati equation, Eq. ( 6$)$, and substituting the matrix $P$ into Eq. ( 8 ), the control law is

$$
G=\left[\begin{array}{llllll}
1.23 & 4.38-1.01 & 6.26 & 0.02-0.05-0.01 & 0.06
\end{array}\right]
$$

After this control law is implemented on the active stay system, the structure dynamic - including mode shape, natural frequency, and damping ratio-of each mode of the four cases considered are shown in Table 1 and Table 2. It is seen that the mode shapes of the stayed and stiffened types are almost the same as those

Table 1 Natural Frequencies $(f)$ and Damping Ratios ( $\zeta)$ of Four Types Considered

\begin{tabular}{|c|ll|ll|ll|ll|}
\hline \multirow{2}{*}{ Mode } & \multicolumn{2}{|l|}{ Original type } & \multicolumn{2}{|l|}{ Stayed type } & \multicolumn{2}{|l|}{ Stiffened type } & \multicolumn{2}{|l|}{ Active type } \\
\cline { 2 - 8 } & $\mathrm{f}(\mathrm{Hz})$ & $\zeta(\%)$ & $\mathrm{f}(\mathrm{Hz})$ & $\zeta(\%)$ & $\mathrm{f}(\mathrm{Hz})$ & $\zeta(\%)$ & $\mathrm{f}(\mathrm{Hz})$ & $\zeta(\%)$ \\
\hline 1 & 2.20 & 0.58 & 7.16 & 0.21 & 6.95 & 0.58 & 6.39 & 42.6 \\
2 & 17.3 & 0.75 & 17.6 & 0.73 & 54.6 & 0.75 & 16.6 & 21.7 \\
3 & 50.7 & 0.60 & 50.7 & 0.60 & 160 & 0.60 & 50.3 & 3.40 \\
4 & 108 & 0.74 & 108 & 0.74 & 340 & 0.74 & 108 & 1.86 \\
\hline
\end{tabular}


Table 2 Eigenvectors of Four Types Conidered

\begin{tabular}{|c|c|c|c|c|}
\hline Mode & Original type & Stayed type & Stiffened type & Active type \\
\hline 1 & $\begin{array}{l}1 \\
-0.098 \mp 0.000 \mathrm{j} \\
0.321 \mp 0.000 \mathrm{j} \\
-0.075 \neq 0.000 \mathrm{j}\end{array}$ & $\begin{array}{l}1 \\
-0.067 \mp 0.000 \mathrm{j} \\
0.424 \neq 0.001 \mathrm{j} \\
-0.085 \neq 0.000 \mathrm{j}\end{array}$ & $\begin{array}{l}1 \\
-0.098 \mp 0.000 \mathrm{j} \\
0.321 \mp 0.000 \mathrm{j} \\
-0.076 \neq 0.001 \mathrm{j}\end{array}$ & $\begin{array}{l}1 \\
-0.088 \pm 0.019 \mathrm{j} \\
0.355 \pm 0.066 \mathrm{j} \\
-0.079 \neq 0.059 \mathrm{j}\end{array}$ \\
\hline 2 & $\begin{array}{l}-0.366 \pm 0.001 \mathrm{j} \\
0.345 \pm 0.001 \mathrm{j} \\
1 \\
-0.054 \mp 0.000 \mathrm{j}\end{array}$ & $\begin{array}{l}-0.444 \pm 0.002 j \\
0.358 \pm 0.001 j \\
1 \\
-0.049 \mp 0.000 j\end{array}$ & $\begin{array}{l}-0.366 \pm 0.001 \mathrm{j} \\
0.345 \pm 0.001 \mathrm{j} \\
1 \\
-0.054 \neq 0.000 \mathrm{j}\end{array}$ & $\begin{array}{l}-0.111 \neq 0.980 \mathrm{j} \\
0.303 \pm 0.177 \mathrm{j} \\
1 \\
-0.073 \neq 0.058 \mathrm{j}\end{array}$ \\
\hline 3 & $\begin{array}{l}-0.142 \pm 0.002 j \\
1 \\
-0.891 \neq 0.005 j \\
-0.527 \neq 0.004 j\end{array}$ & $\begin{array}{l}-0.145 \neq 0.002 j \\
1 \\
-0.890 \neq 0.005 j \\
-0.527 \pm 0.004 j\end{array}$ & $\begin{array}{l}-0.142 \pm 0.002 j \\
1 \\
-0.891 \neq 0.005 j \\
-0.527 \pm 0.004 j\end{array}$ & $\begin{array}{l}-0.091 \neq 0.797 j \\
1 \\
-0.912 \pm 0.336 j \\
-0.541 \neq 0.183 j\end{array}$ \\
\hline 4 & $\begin{aligned} & 0.127 \pm 0.000 j \\
& 0.554 \neq 0.004 j \\
&-0.260 \neq 0.003 j \\
& 1\end{aligned}$ & $\begin{array}{l}0.127 \neq 0.000 j \\
0.554 \neq 0.004 j \\
-0.260 \neq 0.003 j \\
1\end{array}$ & $\begin{array}{l}0.127 \pm 0.001 \mathrm{j} \\
0.554 \mp 0.004 \mathrm{j} \\
-0.260 \pm 0.003 \mathrm{j} \\
1\end{array}$ & $\begin{array}{l}0.166 \pm 0.159 j \\
0.541 \mp 0.055 j \\
-0.262 \neq 0.007 \mathrm{j} \\
1\end{array}$ \\
\hline
\end{tabular}

of the original type. Besides, the natural frequencies of the first mode is stayed type and all modes in stiffened type are rising. It is remarkable that the damping ratio of each mode is not changed by fixed stays auxiliary and direct stiffness increment. However, the structure dynamic of the active type is different from that of the original type. The damping ratio is increased and the real parts of the eigenvalues are evidently moved toward the left in complex plane ${ }^{14}$. From the basic concept of structure dynamics, one knows that the active type will be a superior to the other three types in terms of vibration reduction.

\section{1 Response to Impact Load}

A ship subjected to an impact load is considered herein. Assume that the initial states of the ship body induced by this load are as follows :

$$
\dot{x}_{s}(0)=1 \mathrm{~m} / \mathrm{sec}, x_{s}(0)=\dot{\theta}_{s}(0)=\theta_{s}(0)=0
$$

where $\dot{x}_{s}$ and $x_{s}$ denote the lateral translation velocity and displacement, $\dot{\theta}_{s}$ and $\theta_{s}$ denote the angular velocity and displacement of rotation. Then, the mast will be excited by the movement of the fundation.

By using the control law, described in Eq. (29), Fig. 5 shows the comparisons of the four cases considered for the response of the slope displacement at the top, DOF of $x_{2}$. In the active type, one observes that the $x_{2}$ response will decay quickly to steady state. Assume that the limited value for tracking radar operation is specified by 0.5 milirad. The results show that the decay period is within $0.1 \mathrm{sec}$, which is less than $\frac{1}{500}$ of that of the original type, $\frac{1}{100}$ of that of the stayed type, and $\frac{1}{60}$ of that of the stiffened type. This indicates that the active type has the best performance for slope motion reduction among those types compared which were subjected to impact loads. The accelerations of $x_{1}$, and $x_{2}$ of each type are computed and represented in Fig. 6, and 7. It is surprising that the acceleration response of $x_{1}$ is magnified in both the fixed stay and the stiffness enlarged type. This is a fatal drawback in mast design. However, those acceleration responses in the active type decay rapidly and vanish within a small period of time. By other numerical analysis, we observe that
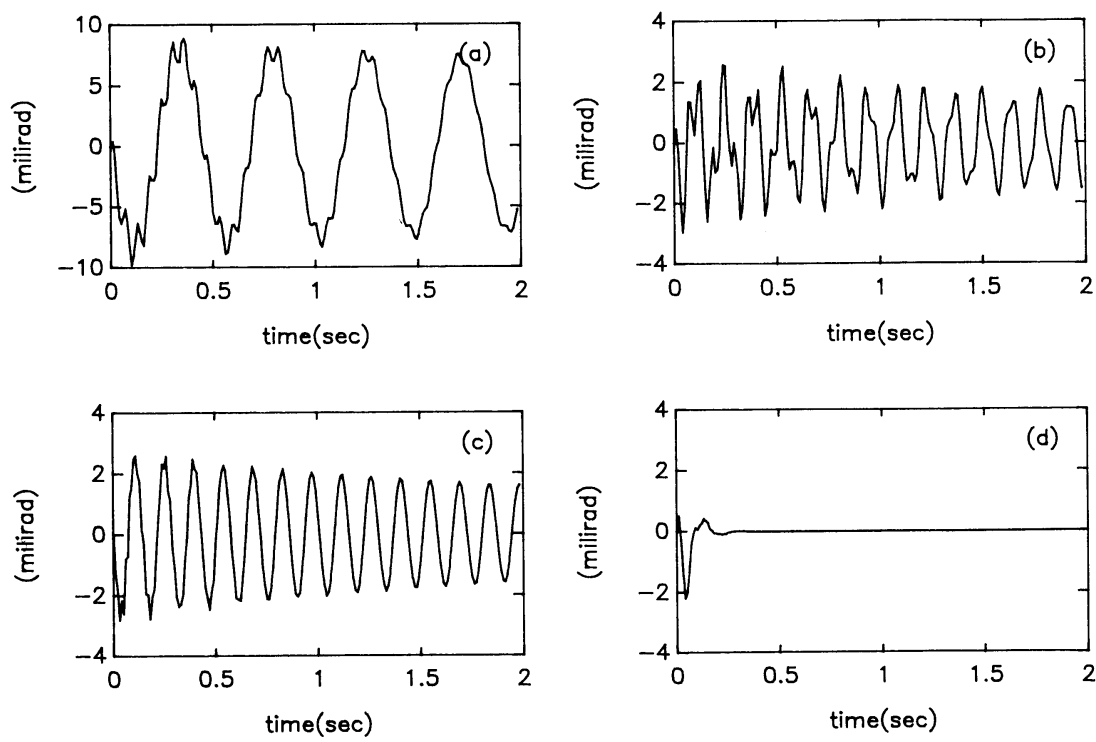

Fig. $5 x_{2}$ Response Due to Impact Load: (a) Original Type; (b) Stayed Type; (c) Stiffened Type; (d) Active Type. 

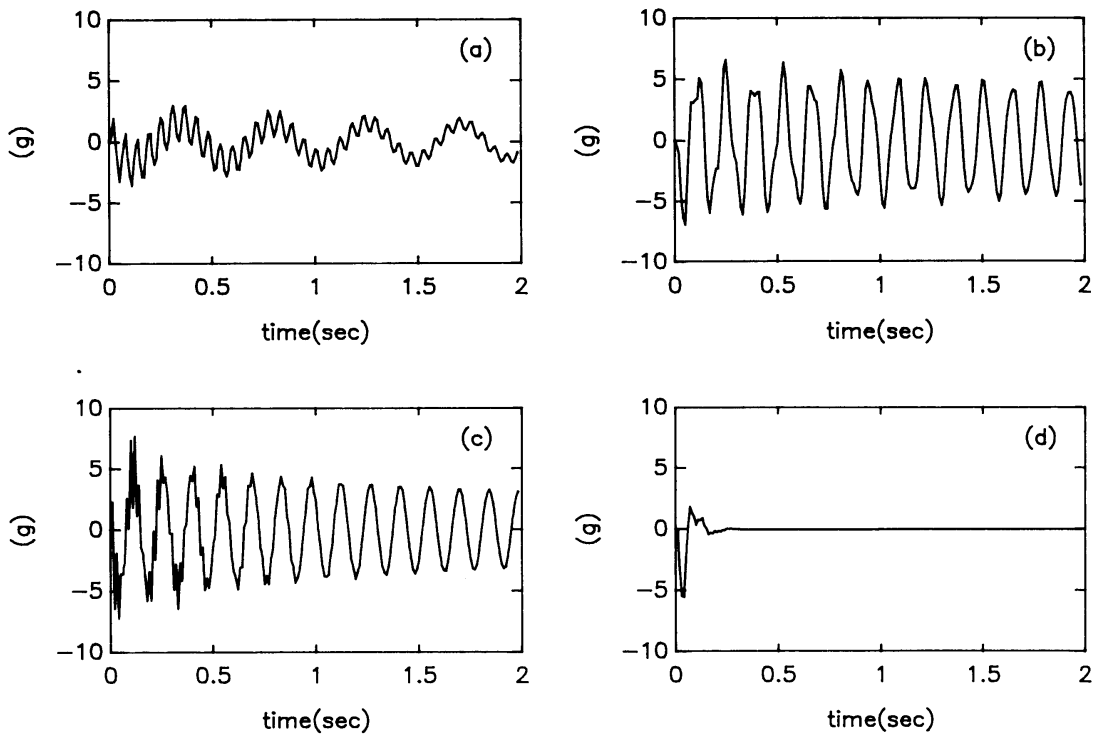

Fig. 6 $\quad \ddot{x}_{1}$ Response Due to Impact Load: (a) Original Type; (b) Stayed Type; (c) Stiffened Type; (d) Active Type.
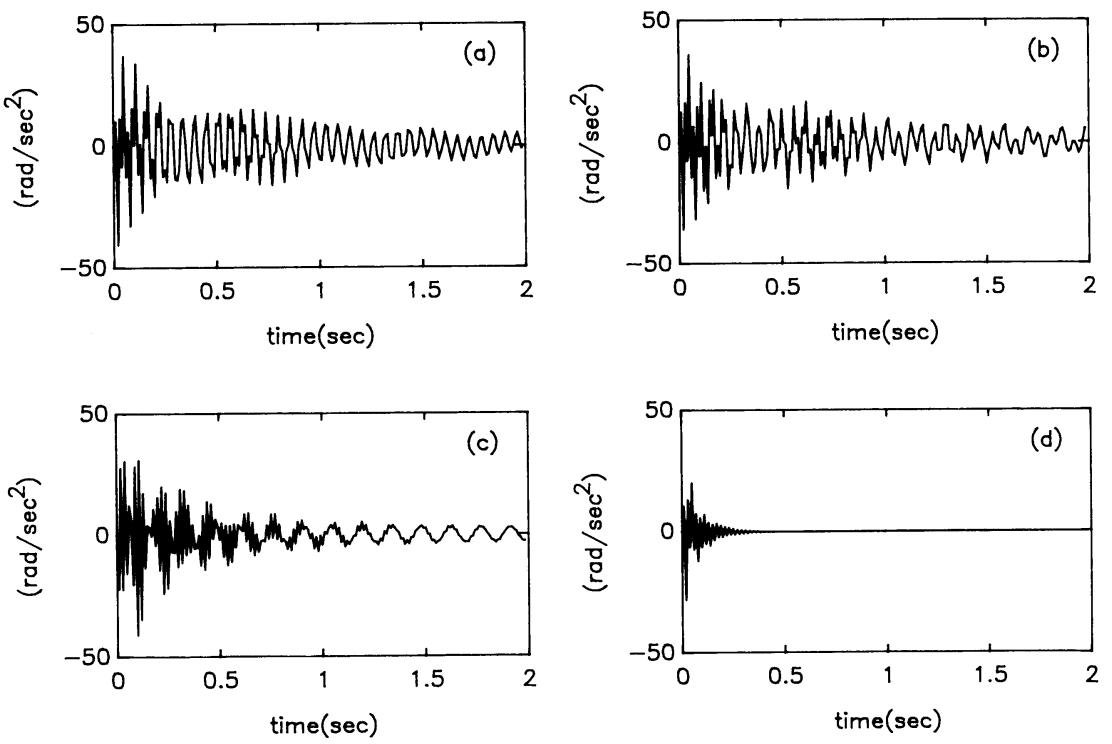

Fig. $7 \quad \ddot{x}_{2}$ Response Due to Impact Load: (a) Original Type; (b) Stayed Type; (c) Stiffened Type;

(d) Active Type.

there is not an obvious difference in the results even if the damping ratios of the polemast structure are assumed to be changed within a reasonable range. The displacement and control force of the actuator are shown in Fig. 8. The required control force is a impulse form. The peak of which appears at the starting point approximately $60 \mathrm{kN}$ and decays quickly.

\section{2 Response to Random Wind Load}

Assume that the external load on the mast by wind gust is considered and the mast deflection induced by this wind gust is much larger than that induced by ship motion. A sphere radar mask with $2 \mathrm{~m}$ diameter is located at the top of the mast. For structures consisting of spherical and cylindrical members of small diameter, 
the wind load can be described as

$$
f_{e}(y, t)=\frac{1}{2} A_{p}(y) \rho_{a} c_{d} v_{w}^{2}(y, t)
$$

where $A_{p}(y)$ is the projection area of structures, $\rho_{a}$ is the density of air, $c_{d}$ is the drag coefficient, and $v_{w}$ is the wind speed. In this example, parameters are chosen as $\rho_{a}=1.226 \mathrm{~kg} / \mathrm{m}^{3}, c_{d}=1.2$ for mast body and 0.4 for radar mask. Assume that wind velocity is a random process. It is indicated that the wind load is also a random process. Thus, the load and speed can be split into mean and fluctuating components

$$
\begin{aligned}
& f_{e}(y, t)=\bar{f}_{e}(y)+\tilde{f}_{e}(y, t) \\
& v_{w}(y, t)=\bar{v}_{w}(y)+\tilde{v}_{w}(y, t)
\end{aligned}
$$

where $\bar{f}_{e}(y)$ and $\bar{v}_{w}(y)$ are mean components, $\tilde{f}_{e}(y, t)$ and $\tilde{v}_{w}(y, t)$ are fluctuating components. By neglecting the term of $\tilde{v}_{w}^{2}$, the relationship of the spectral density function between the fluctuating load and velocity, $S_{f_{e}}$ and $S_{v_{w}}$, is

$$
S_{f e}(y, \omega)=\left(A_{p} \rho_{a} c_{d} \bar{v}_{w}(y)\right)^{2} S_{v_{w}}(y, \omega)
$$

By considering the expected lifetime and the altitude of the mast, mean wind speed with uniform distribution, $\bar{v}_{w}=20.3 \mathrm{~m} / \mathrm{sec}$, is assumed. $S_{v_{w}}(\omega)$ is given by ${ }^{15}$

$$
S_{v_{w}}(\omega)=\frac{0.02 \tilde{\omega}}{(2+\tilde{\omega})^{5 / 6}}
$$

where $\tilde{\omega}$ is a normalized frequency, defined by

$$
\tilde{\omega}=\frac{\omega L}{2 \pi \bar{v}_{w}(10)}
$$

$L$ is a representative-length scale for the turbulence, given as $1200 \mathrm{~m}, \bar{v}_{w}(10)$ is the mean wind velocity at 10 $\mathrm{m}$ height, taken as $20 \mathrm{~m} / \mathrm{sec}$. The curve of $S_{v_{w}}(\omega)$ is shown in Fig. 9. From Eq. (26), Eq. (27), and (34), the spectral density functions of $x_{2}$ of those four types are computed and shown in Fig. 10. It is seen that several high peaks exist in original, stayed, and stiffened types. In the original type, the magnitude of the highest peak is $2 \times 10^{-5} \mathrm{rad}^{2}-\mathrm{sec}$ at frequency $13.8 \mathrm{~Hz}$. In the stayed and stiffened types, that peak is moved to the right, at $20 \mathrm{~Hz}$, but the shape is also very sharp. However, that peaks are suppressed and the response becomes smooth in the active type. This comparison shows that the effect of the resonance suppressing is best by the active stay system. Fig. 11 and Fig. 12 show the spectral density functions of accelerations of $x_{1}$ and $x_{2}$ for those four constructions. The results show that the active

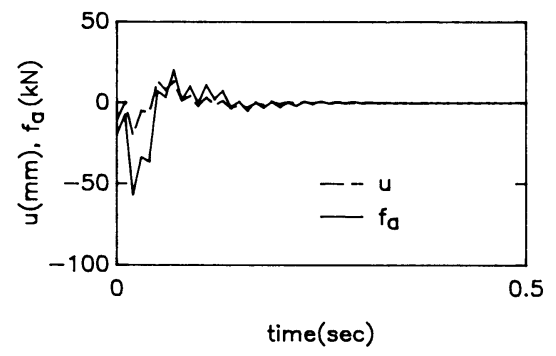

Fig. 8 The Displacement and Force of Actuator for Impulse Load. stay system is also effective for resonance peak suppressing in acceleration response. The standard deviations of those responses are listed in Table 3 . In the active type, the standard variation value of $x_{2}$ response is lower than both the stayed and stiffened types. The value of the active type drops to only $\frac{1}{100}$ of that of the

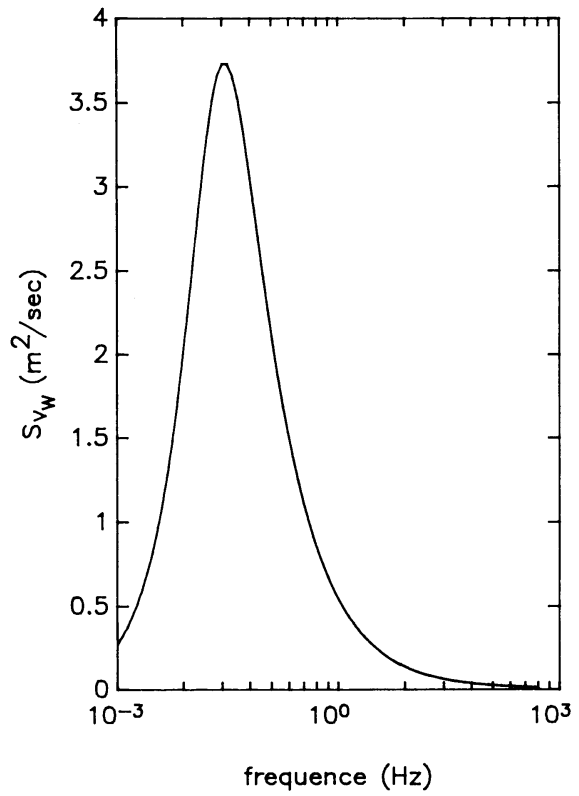

Fig. 9 Spectral Density Function of Wind-gust Speed for Example.

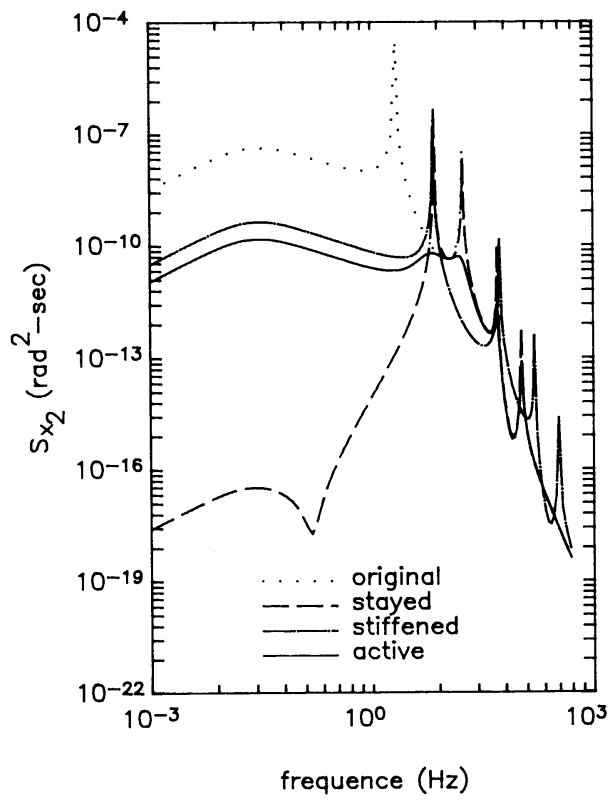

Fig. 10 Spectral Density Function of $x_{2}$ Due to Random Wind-gust Load. 


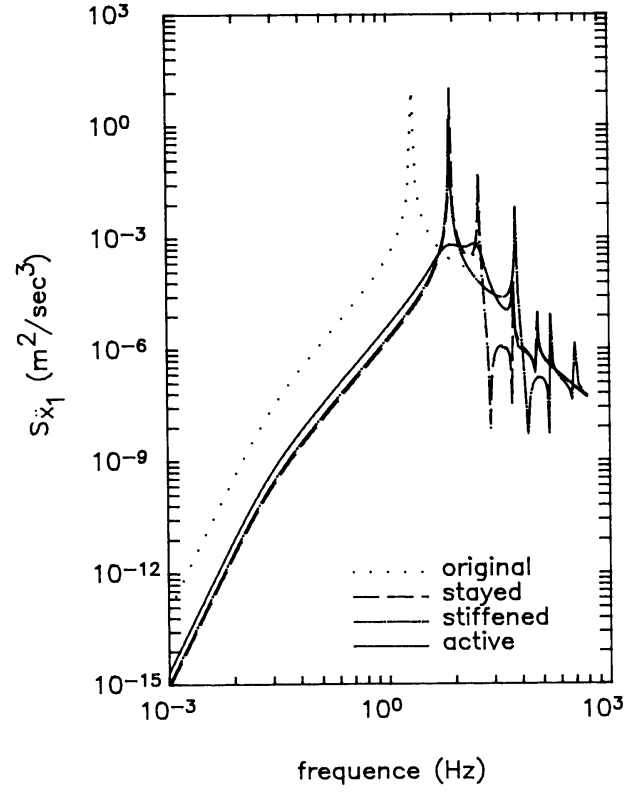

Fig. 11 Spectral Density Function of $\ddot{x}_{1}$ Due to Random Wind-gust Load.

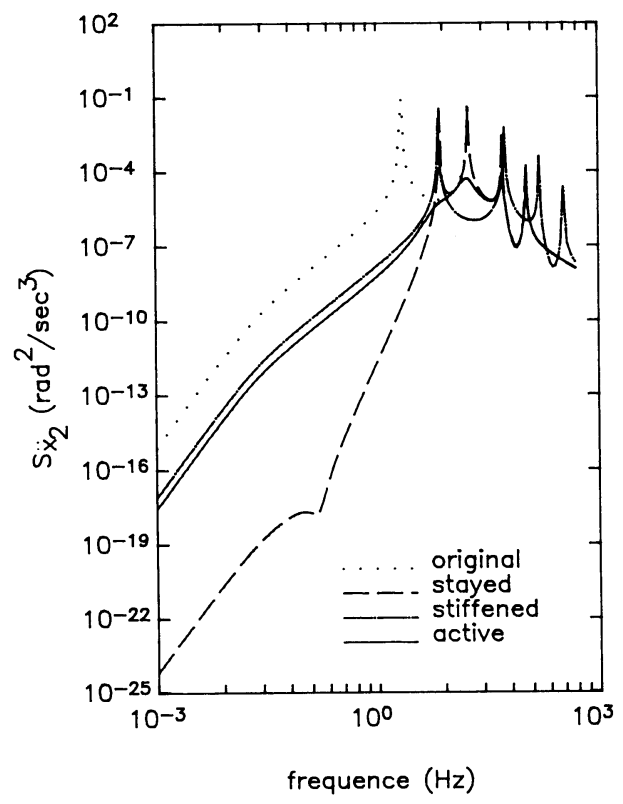

Fig. 12 Spectral Density Function of $\ddot{x}_{2}$ Due to Random Wind-gust Load.

original type and thus proves itself highly effective in reducing slope under random wind load conditions. About the acceleration response of $x_{1}$ and $x_{2}$, the standard variations of the stayed type are larger than those of the original type. This indicates that fixed stay auxiliary may enlarge the acceleration under random
Table 3 Standard Variation Values of Four Types Considered

\begin{tabular}{|l|c|cr|cr|cr|}
\hline & Original type & \multicolumn{2}{|c|}{ Stayed type } & Stiffened type & \multicolumn{2}{|c|}{ Active type } \\
\cline { 2 - 7 } & $(1)$ & $(2)$ & $\frac{(2)}{(1)}$ & $(3)$ & $\frac{(3)}{(1)}$ & $(4)$ & $\left(\frac{4}{(1)}\right)$ \\
\hline$\sigma_{\mathrm{x}_{2}}(\mathrm{rad})$ & $7.05 \times 10^{-3}$ & $1.55 \times 10^{-3}$ & $23 \%$ & $3.32 \times 10^{-4}$ & $4.7 \%$ & $7.62 \times 10^{-5}$ & $1 \%$ \\
$\sigma_{\ddot{\mathbf{x}}_{1}}\left({ }^{\mathrm{m}} / \mathrm{sec}^{2}\right)$ & 3.71 & 6.73 & $181 \%$ & 1.07 & $29 \%$ & 0.25 & $6.7 \%$ \\
$\sigma_{\ddot{\mathbf{x}}_{2}}\left({ }^{\left(\mathrm{rad} / \mathrm{sec}^{2}\right)}\right.$ & 0.51 & 0.66 & $129 \%$ & 0.48 & $94 \%$ & 0.085 & $17 \%$ \\
\hline
\end{tabular}

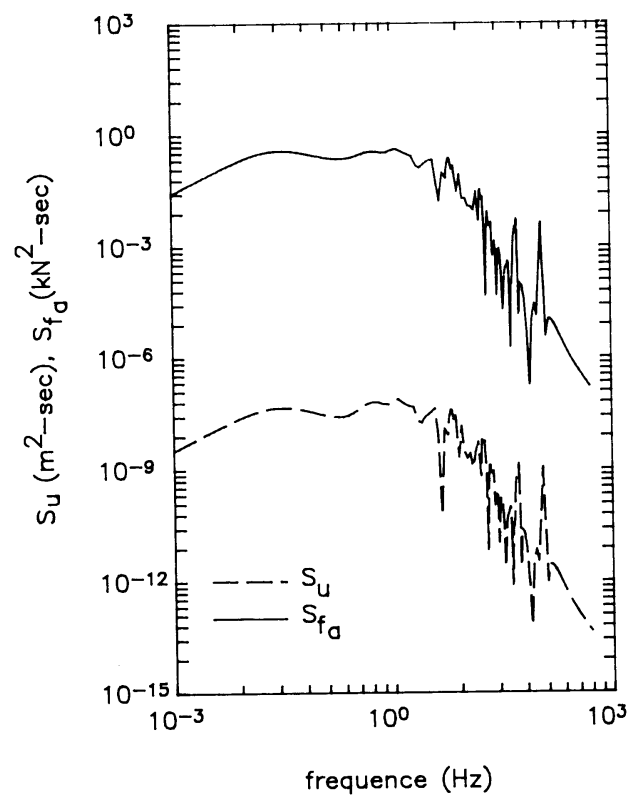

Fig. 13 Spectral Density Function of the Displacement and Force of Actuator.

wind load. In addition, the decrement of these values of the stiffened type with respect to the original type are limited. However, good acceleration alleviation of both lateral translation and slope in the case of mast with the active system is shown. The spectral density functions of displacement and active force of the actuator are shown in Fig. 13. That the active system is feasible is demonstrated by the fact that there are no high peaks over the entire range of frequencies.

\section{Summary and Discussion}

An study of vibration control of the mast in warships by active control system is presented. Since the slope motion at the top of the mast is the important part to be suppressed, a single active stay system is designed to offer the active force to the top of mast. The interaction between the flexibility of the mast and the stay is included in the analysis. In the paper, two typical external excitations - impact load and random 
dynamic load are considered. For each type of load, a cost function is defined to measure the effect of the vibration reduction of the mast and power consumption of the active system. It is shown that the optimal control functions for both typical loads are identical. Then the minimization of the cost function is analyzed to obtain the optimal control function that the actuator should follow. By applying the optimal control law, which is obtained by appropriate selection of the weighting factors of the performance index function, the damping of the active stay type will increase evidently.

In order to demonstrate the effect of this scheme, four types of constructions (polemast without auxiliary, direct stiffness increment, with fixed stay auxiliary, and with active stay system) subjected to both types of load, are computed and compared in numerical examples. The results show that the active stay system has the fastest decay property among those methods for the mast subjected to ship inpulse motion. For random wind load, it has superior effect of peak suppressed in spectral density response than other three methods and the standard variations much lower than other cases compared. In addition, the lateral translation and slope accelerations at the top of the mast reduced by the active stay system is also the most effective by ship impulse motion and random wind load. From the active force consumed in examples, it is shown that the active stay system with optimal control function is a powerful and feasible scheme for mast vibration reduction.

\section{Acknowledgement}

The research of this paper was supported by the National Science Council of the Republic of China under Grant No. NSC 81-0403-E-002-01.

\section{References}

1) Long, G., "Vibration on Marine Structures", Seminar on Mechanical Engineering in a Marine
Environment, Australia, June 1983.

2) Pegg, N. G., "Destroyer Mast Vibration Analysis Using Finite Elements", Tenth Canadian Congress of Applied Mechanics, Canada, June 1985.

3) Robinsoon, D. C. and Antonides, G. P., "Uss Tripoli (Lph 10) Underway Vibration Trials", NTIS Report DTMB-2306, Oct. 1966.

4) "Mechanical Vibrations of Shipboard Equipment (Type I -Environmental and Type II-Internally Excited)", Military Standard MIL-STD-167-1, Naval Ship Systems Command, USA, May 1974.

5) "Shock Design Criteria for Surface Ships", $N A V$ SEA-0908-LP-000-3010, Naval Sea Systems Command, USA, May 1976.

6) Losee, L. K., "Stayed Polemast", Design Data sheat DDS1702-1, Bureau of Ships, USA, Sept. 1957.

7) "Four-Legged Mast", Design Data Sheet DDS1701-3, Bureau of Ships, USA, May 1960.

8) Meirovitch, L. and Kwak, M. K., "Control of Spacecraft with Multi-Targeted Flexible Antennas", The Journal of the Astronautical Sciences, Vol. 38, No. 2, 1990.

9) Madany, M. M. E. and Razaz, Z. S. E., "Performance of Actively Suspended Cabs in Highway Trucks-Evaluation and Optimization", Journal of Sound and Vibration, Vol. 126, No. 3, 1988.

10) Samali, B., Yang, J. N., and Liu, S. C., "Active Control of Seismic-Excited Buildings", Journal of the Structural Engineering, ASCE, Vol.109, No. 1, 1983.

11) Hsueh, W. J. and Lee, Y. J., "Study of the Vibration Reducing of Mast by Active Control Method", Journal of the SNAME, ROC, Vol.9, No. 1, June 1990.

12) Clough, R. W. and Penzien, J., Dynamics of Structures, McGraw-Hill, 1975.

13) Kwakernaak, H. and Sivan, R., Linear Optimal Control System, John Wiley \& Sons, 1972.

14) Meirovitch, L., Elements of Vibration Analysis, 2nd ed., McGraw-Hill, 1986.

15) Brebbia, C. A. and Walker, S., Dynamic Analysis of Offshore Structures, Butterworth, UK, 1979. 